\title{
Observaciones con MEB de cinco especies de Erysiphales en plantas ornamentales y frutales en el Estado de México, México
}

\author{
SEM observations of five Erysiphales species on ornamental and fruit plants \\ in the State of Mexico, Mexico
}

\begin{abstract}
Juan Manuel Tovar-Pedraza', Greta Hanako Rosas-Saito², Mayra Teresa García-Ruiz ${ }^{3}$, Moisés Camacho-Tapia ${ }^{4}$, Santos Gerardo Leyva-Mir ${ }^{5}$

${ }^{1}$ Centro de Investigación en Alimentación y Desarrollo, A. C., Coordinación Culiacán, Laboratorio de Fitopatología. Carretera El Dorado Km 5.5, Campo el Diez, 80110, Culiacán, Sinaloa, México.

${ }^{2}$ Instituto de Ecología, Red de Estudios Moleculares Avanzados. Carretera antigua a Coatepec 351, El Haya, Xalapa, 91070, Veracruz, México.

${ }^{3}$ Universidad Autónoma Chapingo, Centro de Educación Continua, Texcoco, 56230, Estado de México, México.

${ }^{4}$ Universidad Autónoma Chapingo, Laboratorio Nacional de Investigación y Servicio Agroalimentario y Forestal, Texcoco, 56230, Estado de México, México.

${ }^{5}$ Universidad Autónoma Chapingo, Departamento de Parasitología Agrícola, Texcoco, 56230, Estado de México, México.
\end{abstract}

\section{RESUMEN}

Antecedentes: Los patrones de paredes terminales y externas de conidios observados en microscopia electrónica de barrido (MEB) combinados con caracteres analizados en microscopia de luz (ML) son una herramienta poderosa para la identificación de especies de anamorfos de Erysiphales.

Objetivo: Realizar un análisis morfológico mediante MEB de los anamorfos de Erysiphales presentes en plantas de onagra (Oenothera fruticosa), mercadela (Calendula officinalis), hortensia (Hydrangea macrophylla), margarita (Leucanthemum vulgare) y limón (Citrus limon).

Métodos: La caracterización morfológica de los especímenes de Erysiphales se realizó mediante MEB y se complementó usando ML.

Resultados y conclusiones: La examinación mediante MEB permitió la caracterización de conidióforos, conidios, apresorios hifales, hifas, y tubos germinativos de Erysiphe sp., Podosphaera xanthii, Pseudoidium hortensiae, Golovinomyces macrocarpus y Erysiphe quercicola. Para nuestro conocimiento, este es el primer reporte de Golovinomyces macrocarpus causando cenicilla en L. vulgare en México.

Palabras clave: Erysiphe sp., Podosphaera xanthii, Pseudoidium hortensiae, Golovinomyces macrocarpus, Erysiphe quercicola

\section{ABSTRACT}

Background: The patterns on outer and end walls of conidia observed in scanning electron microscopy (MEB) combined with light microscopy (ML) analysis are a powerful tool for the identification of Erysiphales anamorph species.

Objective: To perform a morphological analysis by MEB of Erysiphales anamorphs present on sundrops (Oenothera fruticosa), common marigold (Calendula officinalis), bigleaf hydrangea (Hydrangea macrophylla), oxeye daisy (Leucanthemum vulgare) and lemon (Citrus limon) plants.

Methods: The morphological characterization of Erysiphales specimens was performed by SEM and complemented using ML.

Results and conclusions: The examination by MEB allowed the characterization of conidiophores, conidia, hyphal appressoria, hyphae, and germ tubes of Erysiphe sp., Podosphaera xanthii, Pseudoidium hortensiae, Golovinomyces macrocarpus and Erysiphe quercicola. To our knowledge, this is the first report of Golovinomyces macrocarpus causing powdery mildew on L. vulgare in Mexico.

Keywords: Erysiphe sp., Podosphaera xanthii, Pseudoidium hortensiae, Golovinomyces macrocarpus, Erysiphe quercicola

\section{ARTICLE HISTORY}

Received 19 June 2018 / Accepted 25 October 2018

On line 02 November 2018

\section{CORRESPONDING AUTHOR}

\u Juan Manuel Tovar-Pedraza, juan.tovar@ciad.mx

ORCID: 0000-0002-8560-959X 


\section{INTRODUCCIÓN}

Las cenicillas (Erysiphales) representan un grupo común de hongos patógenos obligados de plantas, los cuales tienen distribución cosmopolita y pueden ser fácilmente reconocidos por los síntomas y signos típicos que producen (Braun y Cook, 2012). Estos síntomas y signos se pueden observar como crecimiento polvoso blanco-grisáceo principalmente sobre las superficies de las hojas, pero también se pueden encontrar en tallos e inflorescencias (Gleason et al., 2009).

Los primeros estudios llevados a cabo por Plumb y Turner (1972), Hammett (1977) y Gorter (1987) en los que se examinaron caracteres morfológicos de especies de Erysiphales usando microscopia electrónica de barrido (MEB), sólo describieron el patrón superficial de conidios para algunos géneros y especies, pero no presentaron suficiente información para usar dichos caracteres dentro de una clasificación taxonómica. Conviene destacar que el estudio realizado por Cook et al. (1997) fue el primer trabajo que registró el patrón superficial de conidios y de septos de diversos géneros y especies de Erysiphales con implicaciones taxonómicas. Estos patrones superficiales observados en MEB sirven como apoyo para identificación cuando los caracteres cruciales no son claros con microscopia de luz (ML), además de proveer una herramienta adicional útil para la identificación de anamorfos de cenicillas desconocidas (Braun et al., 2002; Braun y Cook, 2012). En las últimas décadas, las investigaciones y avances llevados a cabo con el uso de microscopia electrónica de barrido y biología molecular influenciaron de manera significativa el desarrollo del sistema taxonómico de los Erysiphales y su interpretación filogenética (Braun et al., 2002).

En general, existen cuatro patrones de las paredes terminales, 11 patrones de las paredes externas de conidios turgentes, y nueve patrones de conidios arrugados, los cuales pueden ser observados principalmente mediante análisis en MEB (Cook et al., 1997; Braun et al., 2002; Braun y Cook, 2012). Cabe resaltar, que la mayoría de los principales géneros de holomorfos de Erysiphales se pueden identificar mediante los patrones superficiales observados en MEB, excepto Cystotheca, sin embargo, este género puede ser fácilmente identificado por las setas características que se observan sobre el micelio (Braun y Cook, 2012). Por su parte, Braun (1999) y Braun et al. (2002) resaltaron la necesidad de realizar un mayor número de estudios que involucren la exploración de regiones donde se conozca poco sobre las cenicillas, asi como examinaciones detalladas de los anamorfos usando la combinación de $M L$ y MEB, con la finalidad de extender el conocimiento sobre los Erysiphales y su taxonomía. Al respecto, Braun y Cook (2012) complementaron la información de ML con estudios de MEB, lo cual permite diferenciar a los géneros y a diversas especies de anamorfos de Erysiphales, sin la necesidad de caracterizar los casmotecios del teleomorfo o estado sexual. No obstante, existen complejos de especies de Erysiphales que únicamente pueden ser identificadas o distinguidas mediante la combinación de caracterización morfológica y análisis de secuencias de ADN (Braun y Cook, 2012; Bereczky et al., 2015; Scholler et al., 2016; Pastirčáková et al., 2016; Bradshaw et al., 2017).

Existen algunos estudios previos (Félix-Gastélum et al., 2005, 2007, 2011, 2013, 2014; Domínguez-Serrano et al., 2016; Tovar-Pedraza et al., 2016; Camacho-Tapia et al., 2018) en los cuales se analizaron las características morfológicas de especies de Erysiphales presentes en México usando MEB. Sin embargo, se necesita un mayor número de estudios que describan a detalle los patrones de la superficie externa y del septo de diversas especies de Erysiphales. Por lo anterior, el objetivo de este estudio fue caracterizar mediante MEB a las especies de Erysiphales presentes sobre plantas de onagra (Oenothera fruticosa), mercadela (Calendula officinalis), hortensia (Hydrangea macrophylla), margarita (Leucanthemum vulgare) y limón (Citrus limon), además, de complementar dicha caracterización con examinaciones en microscopia de luz.

\section{MATERIALES Y MÉTODOS}

\section{Recolección de material}

Durante los años 2013-2016, se recolectaron muestras vegetales con síntomas de cenicillas en plantas de onagra, mercadela, hortensia y margarita localizadas en jardines de Texcoco, Estado de México, México. Las muestras de limón con síntomas y signos de cenicilla se recolectaron en árboles de traspatio localizados en los municipios de Villa Guerrero y Texcoco en el Estado de México, México. Los especímenes representativos de cada planta hospedante se depositaron en el herbario del Departamento de Parasitología Agrícola de la Universidad Autónoma Chapingo con los números de acceso UACH H115-H119. 
Análisis en microscopia de luz (ML)

Para el análisis en microscopia de luz, se realizaron preparaciones semipermanentes a partir de signos del hongo sobre una gota de ácido láctico al $85 \%$ y ligeramente calentada. Las características morfológicas de 100 conidios, 50 conidióforos, 10 apresorios hifales y 10 tubos germinativos se examinaron en un microscopio de luz Eclipse Ni-U (Nikon Corporation, Japón) equipado con una cámara Moticam 580 (Motic, China) para su caracterización cualitativa y cuantitativa. La identificación a nivel de género y especie se realizó siguiendo las claves especializadas para Erysiphales reportadas por Braun y Cook (2012).

\section{Análisis en microscopia electrónica de barrido (MEB)}

Para el análisis en MEB, se fijaron piezas pequeñas de hojas $\left(5 \mathrm{~mm}^{2}\right.$ ) con ligera esporulación, en frascos conteniendo glutaraldehído (Electron Microscopy Science, USA) al $3 \%$ y se almacenaron a $4{ }^{\circ} \mathrm{C}$ por $24 \mathrm{~h}$, para ser procesadas de acuerdo con la metodología reportada por Bozzola y Russell (1992). Las piezas de hojas fijadas se lavaron tres veces en buffer de fosfatos Sorensen $0.1 \mathrm{M}, \mathrm{pH} 7.2$ por $20 \mathrm{~min}$ en cada cambio, se deshidrataron en una serie gradual de etanol $(30,40$, $50,60,70,80,90$ y $96 \%)$ por 20 min, y dos veces en etanol al $100 \%$ por 30 min en cada cambio. Los especímenes se colocaron en una secadora de punto crítico Sandri-780 (Tousimis Research Corporation, USA) con $\mathrm{CO}_{2}$. El material desecado se transfirió a un portamuestras metálico usando una cinta adhesiva doble de cobre y se cubrieron con una capa de oro de $500 \AA$ de grosor en una ionizadora lon Sputter JFC-1100 (Jeol, Japón) por 4 min. Los especímenes se examinaron en un microscopio electrónico de barrido JSM-6390 (Jeol, Japón) operado a una aceleración de voltaje de $10 \mathrm{kV}$ y se tomaron micrografías a magnificaciones entre 600 y $10,000 x$.

\section{RESULTADOS Y DISCUSIÓN}

\section{Síntomas}

En plantas de $O$. fruticosa se observaron inicialmente colonias fúngicas dispersas a manera de parches grisáceos principalmente sobre la superficie adaxial de las hojas (Figura 1A-B). Posteriormente, las colonias se expandieron y coalecieron hasta cubrir la superficie de las hojas casi en su totalidad. En infecciones severas, se observó crecimiento micelial sobre los tallos de las plantas. De acuerdo con Gleason et al. (2009), las plantas de $O$. fruticosa son comúnmente susceptibles a la infección por Erysiphales y los síntomas se presentan típicamente como un crecimiento fúngico blanco-grisáceo sobre la superficie inferior de las hojas. Sin embargo, no hay reportes sobre las especies de Erysiphales que causan esta enfermedad en México.

Con respecto a $C$. officinalis, los signos se observaron a manera de polvo blanco-grisáceo principalmente sobre la superficie adaxial de las hojas (Figura 1C). En infecciones severas, la totalidad de la hoja fue cubierta por los signos del hongo. Cabe mencionar que no se observaron signos del hongo sobre tallos o inflorescencias. Al respecto, Lucero et al. (2007) reportaron síntomas similares en plantas de C. officinalis en Argentina y también indicaron que las hojas afectadas se pueden deformar y marchitar para posteriormente secarse.

Acerca de las plantas de $H$. macrophylla, éstas exhibieron síntomas a manera de parches blanquecinos sobre la superficie adaxial (Figura 1D) y abaxial de las hojas. En infecciones severas, se observó una necrosis del tejido foliar. Estos síntomas coincidieron con los reportados por Woodward y Daughtrey (2001) para el caso de cenicillas infectando Hydrangea spp., además indicaron que, bajo condiciones óptimas para el desarrollo de la enfermedad, se pueden observar manchas marrones-purpuras cubiertas por crecimiento fúngico blanco.

En cuanto a los síntomas en plantas de L. vulgare, éstos se presentaron inicialmente como lesiones cloróticas en la superficie adaxial de las hojas, las cuales posteriormente se cubrieron de crecimiento fúngico blanco (Figura 1E). De acuerdo con Braun y Cook (2012), varias especies de Leucanthemum pueden ser infectadas con cenicilla, incluyendo a L. maximum, $L$. monspeliense y L. vulgare.

En árboles de limón se observó que todas las partes verdes (hojas, ramitas, brotes y frutos) presentaron síntomas. Éstos inician como pequeñas colonias de micelio blanco sobre la superficie de los tejidos y, posteriormente, pueden llegar a cubrir completamente los tejidos con abundante producción de estructuras asexuales del hongo. Los tejidos mayormente infectados son los que se localizaron en la parte inferior y central de la copa del árbol, coincidiendo con la parte más sombreada. Las hojas son las partes más comúnmente colonizadas por el hongo tanto en la superficie 
adaxial como en la abaxial, causando en infecciones severas una deformación de hojas y defoliación prematura. Las ramitas infectadas pueden cubrirse casi en su totalidad y exhibir muerte descendente. Los brotes o tejidos jóvenes son afectados severamente y llegan a presentarse deformaciones de hojas, al ser cubiertas completamente por el micelio. Los frutos infectados son cubiertos por micelio y esporulación del hongo casi en su totalidad y en infecciones severas se presenta caída prematura. Estos síntomas concuerdan con los reportados por Holford et al. (2010) para el caso de árboles de mandarina (Citrus reticulata) infectados con E. quercicola en Bután.

\section{Caracterización morfológica}

El análisis en ML de los caracteres morfológicos del hongo infectando $O$. fruticosa mostró conidióforos erectos originando conidios simples; las células base de los conidióforos fueron cilíndricas, midiendo de 24.3-33.8 × 6.3-10.4 $\mu \mathrm{m}$, y seguida por 1-2 células más cortas; los conidios fueron elipsoides, doliiformes y cilíndricos, de 22.4-34.5 × 9.6-15.8 $\mu \mathrm{m}$ (Figura 2A); los tubos germinativos fueron terminales, cortos, y con apresorios conidiales lobados (Figura 2B y 2C). Mientras que, en MEB se observaron apresorios hifales lobados, solitarios (Figura 2D) o en pares opuestos; los conidióforos fueron erectos y originaron conidios
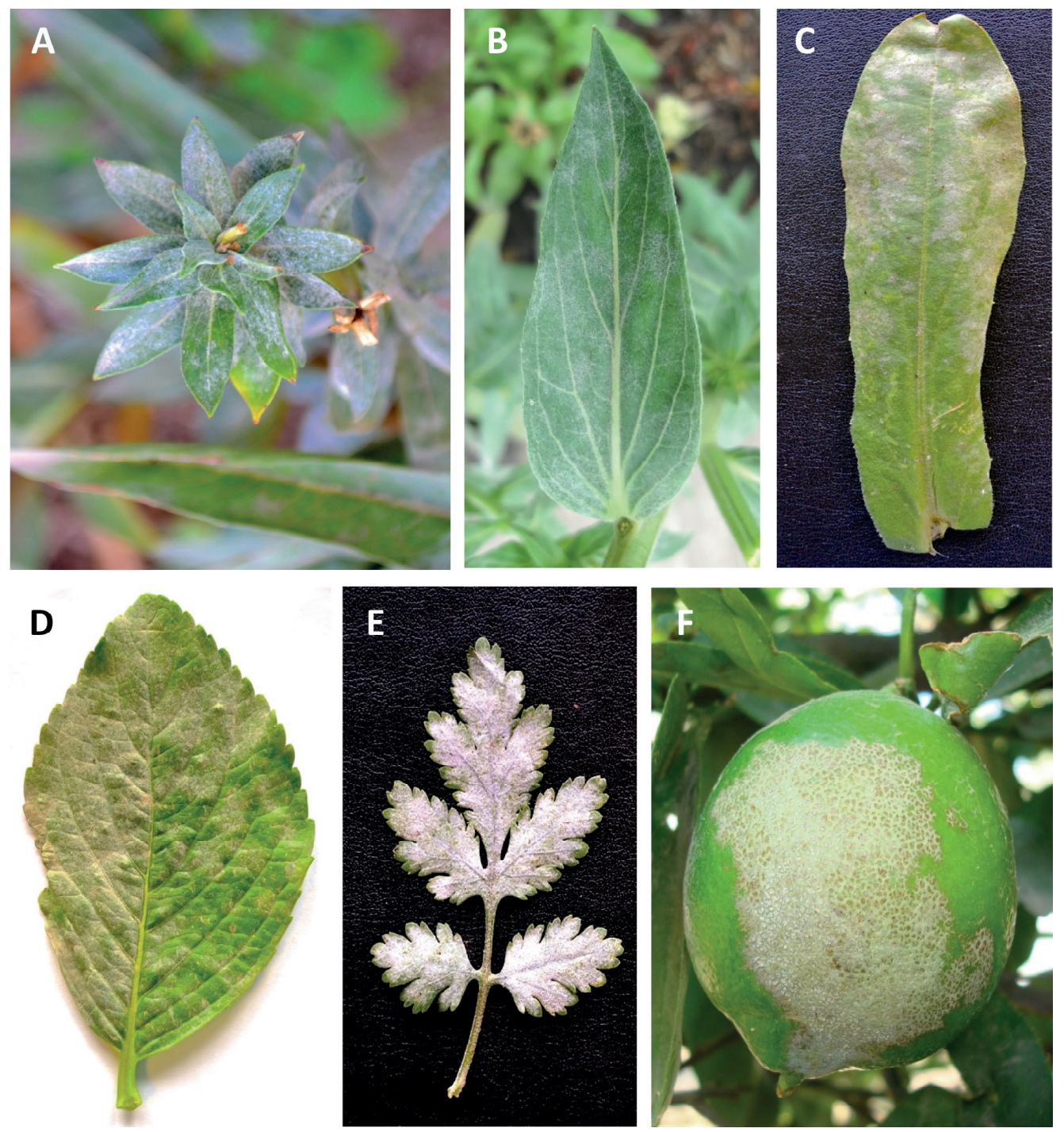

Figura 1. Síntomas y signos de cenicilla en plantas ornamentales y frutales. A-B) Crecimiento fúngico blanco-grisáceo de Erysiphe sp. en hojas de Oenothera fruticosa; C) Hoja de Calendula officinalis con síntomas de cenicilla causados por Podosphaera xanthii; D) Síntomas de cenicilla causados por Pseudoidium hortensiae en hojas de Hydrangea macrophylla. E) Hoja de margarita con crecimiento fúngico sobre la superficie superior causado por Golovinomyces macrocarpus. F) Fruto de limón real cubierto por crecimiento fúngico blanco de Erysiphe quercicola. 
individuales (Figura 2E); los conidios turgentes presentaron pared externa con patrón rugoso (Figura 2F) y pared terminal con patrón fibrilar (Figura 2G). No se observaron casmotecios. Todas las características morfológicas del anamorfo fueron consistentes con las reportadas por Braun y Cook (2012) para el caso de los estados asexuales de Erysiphe howeana y E. alphitoides. Por consiguiente, nuestro estudio no logró distinguir entre ambas especies de Erysiphales mediante el uso de las características morfológicas del anamorfo. Esto coincidió con Bereczky et al. (2015), quienes indicaron que las especies Erysiphe howeana y E. alphitoides en plantas de Oeneothera spp., únicamente pue- den ser distinguidas mediante el análisis de secuencias del ADN ribosómico.

La examinación en MEB realizada al hongo que se encontró infectando tejidos de C. officinalis mostró conidióforos con conidios catenados parcialmente deshidratados mostrando patrón sinuoso en la pared externa (Figuras 3A y B); los apresorios hifales fueron solitarios y en forma de pezón (Figura 3C); los conidios turgentes presentaron pared externa con patrón liso y con superficie de la pared terminal con patrón verticilado (Figura 3D); los conidios exhibieron tubos germinativos más o menos terminales, simples (Figura 3E) o bifurcados (Figura 3F). No se observó la presencia
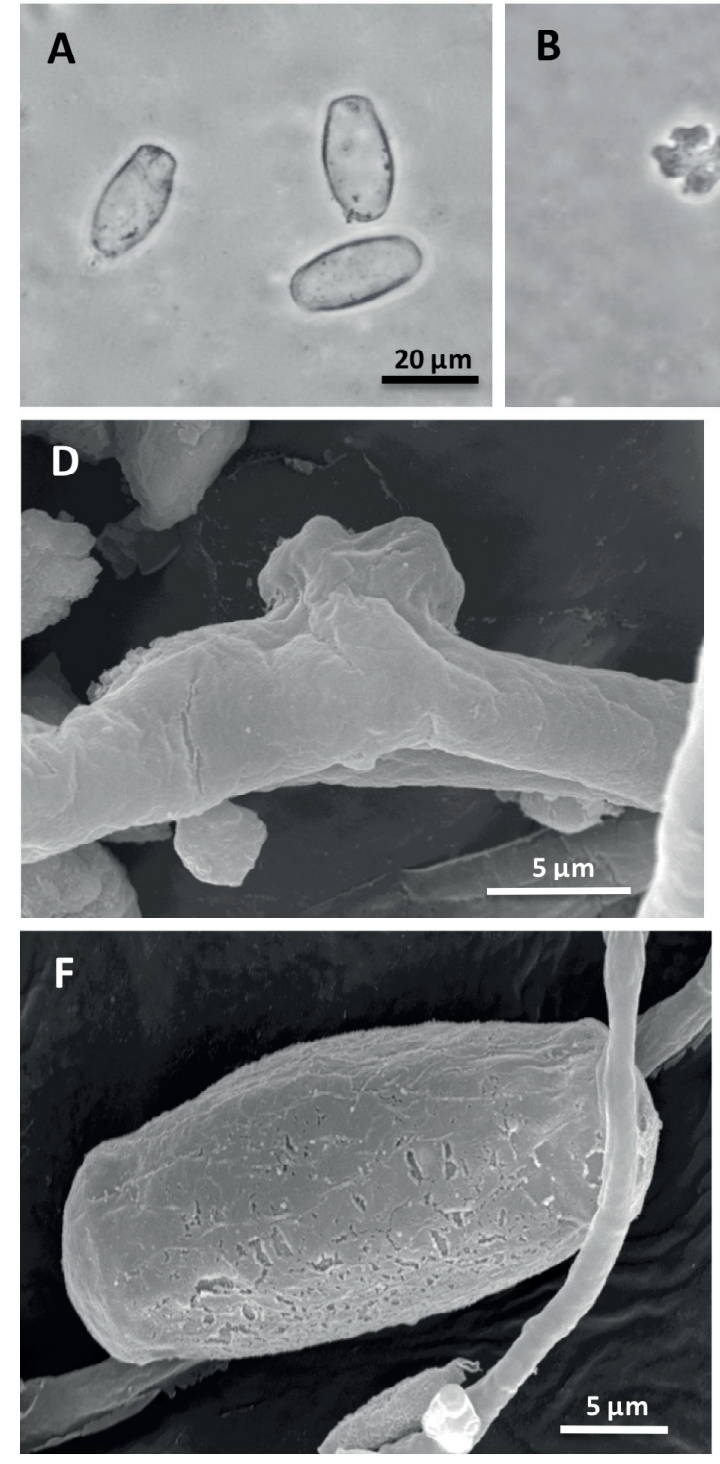
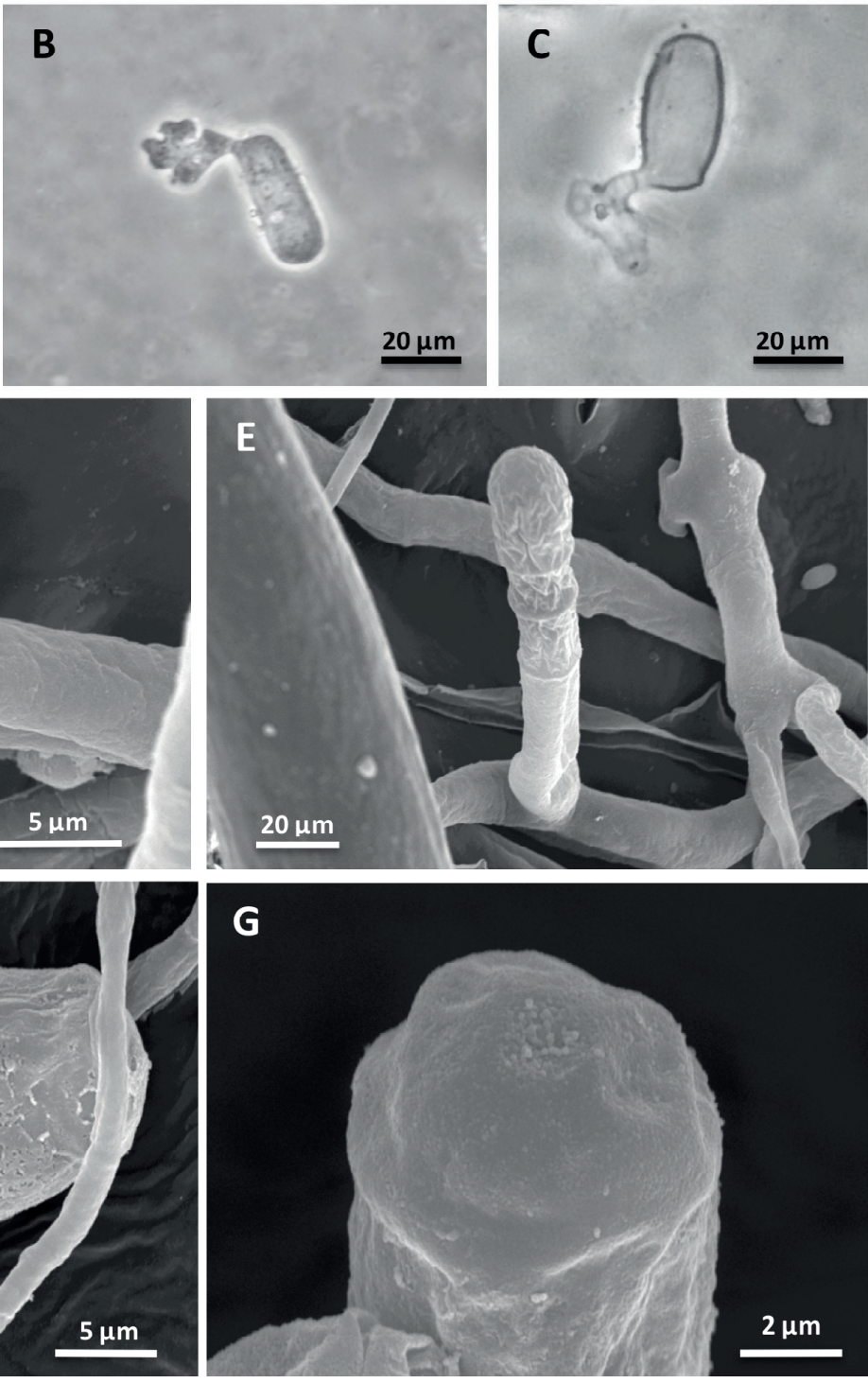

Figura 2. Micrografías en microscopia de luz A-C) y microscopia electrónica de barrido D-G) del estado asexual de Erysiphe sp. sobre hojas de Oenothera fruticosa. A) Conidios. B-C) Conidios germinando y con apresorio conidial lobado. D) Apresorio hifal lobado y solitario. E) Conidióforo con conidio simple. F) Conidio turgente mostrando pared externa con patrón rugoso. G) Pared terminal del conidio con patrón fibrilar. 

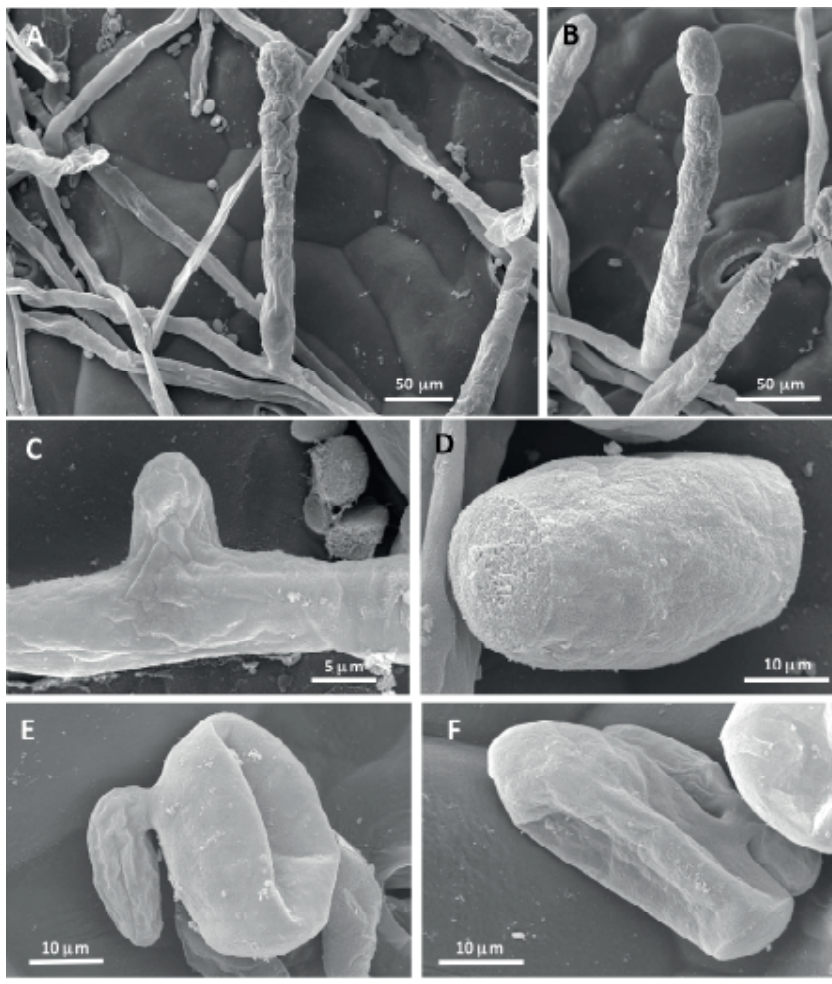

\begin{abstract}
Figura 3. Micrografías en microscopia electrónica de barrido del estado asexual de Podosphaera xanthii. A-B) Conidióforos con conidios catenados (parcialmente deshidratados) mostrando patrón superficial sinuoso. C) Apresorio hifal solitario y en forma de pezón. D) Conidio

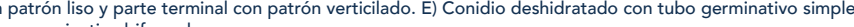
F) Conidio deshidratado con tubo germinativo bifurcado.

de casmotecios. Las características del anamorfo coincidieron ampliamente con las descripciones en MEB de Podosphaera xanthii reportadas sobre calabaza zucchini (Cucurbita pepo) (Félix-Gastélum et al., 2005) y tomatillo (Physalis ixocarpa) (Félix-Gastélum et al., 2007) en México. Por otra parte, el análisis en ML mostró hifas lisas con pared delgada; los apresorios hifales fueron solitarios y en forma de pezón; los conidióforos fueron erectos y originándose de la superficie superio de la célula madre; las células basales de los conidióforos presentaron forma cilíndrica, midiendo 41.6-65.9 ×

conidios fueron catenados, elipsoides-ovoides a doliiformes y midiendo 24.4-36.8 × 14.8-21.1 $\mu \mathrm{m}$. Estos caracteres morfológicos concuerdan con las descripciones reportadas para el estado anamorfico de Podosphaera xanthii (Braun y Cook, 2012). Previamente, Yáñez-Morales et al. (2009) reportaron la presencia del anamorfo de $P$. xanthii sobre $C$. officinalis en México. Asimismo, nosotros no observamos la presencia de casmotecios, en contraste con otros estudios realizados en Argentina (Lucero et al., 2007) e Italia (Garibaldi et al., 2008), donde se ha observado comúnmente la
\end{abstract}

FiguRa 3. Micrografías en microscopia electrónica de barrido del estado asexual de Podosphaera xanthii. A-B) Conidióforos con conidios catenados (parcialmente deshidratados) mostrando patrón superficial sinuoso. C) Apresorio hifal solitario y en forma de pezón. D) Conidio turgente con pared externa con patrón liso y parte terminal con patrón verticilado. E) Conidio deshidratado con tubo germinativo simple. F) Conidio deshidratado con tubo germinativo bifurcado.

de casmotecios. Las características del anamorfo coincidieron ampliamente con las descripciones en MEB de Podosphaera xanthii reportadas sobre calabaza zucchini (Cucurbita pepo) (Félix-Gastélum et al., 2005) y tomatillo (Physalis ixocarpa) (Félix-Gastélum et al., 2007) en México. Por otra parte, el análisis en ML mostró hifas lisas con pared delgada; los apresorios hifales fueron solitarios y en forma de pezón; los conidióforos fueron erectos y originándose de la superficie superior de la célula madre; las células basales de los conidióforos presentaron forma cilíndrica, midiendo 41.6-65.9 × 10.9-13.4 $\mu \mathrm{m}$, seguidas por 2-3 células más cortas; los conidios fueron catenados, elipsoides-ovoides a doliiformes y midiendo $24.4-36.8 \times 14.8-21.1 \mu \mathrm{m}$. Estos caracteres morfológicos concuerdan con las descripciones reportadas para el estado anamorfico de Podosphaera xanthii (Braun y Cook, 2012). Previamente, Yáñez-Morales et al. (2009) reportaron la presencia del anamorfo de P. xanthii sobre $C$. officinalis en México. Asimismo, nosotros no observamos la presencia de casmotecios, en contraste con otros estudios realizados en Argentina (Lucero et al., 2007) e Italia (Garibaldi et al., 2008), donde se ha observado comúnmente la presencia de casmotecios en plantas de $C$. officinalis. 
La examinación en MEB de la especie de hongo infectando $H$. macrophylla, reveló conidios formados individualmente sobre el conidióforo (Figura 4A); los conidios turgentes mostraron patrón rugoso en la pared externa (Figura 4B) y patrón fibrilar en la parte terminal (Figura 4C); los apresorios hifales fueron multilobados, solitarios o en pares opuestos (Figura 4D). Por otra parte, en ML se observaron conidióforos erectos y originándose más o menos en la parte central de la superficie superior de la célula madre y midiendo 53-116 × 8.1-9.7 $\mu \mathrm{m}$; las células basales de los conidióforos fueron cilíndricas, algunas veces ligeramente curvadas, midiendo 16.3-38.6 × 6.2-9.9 $\mu \mathrm{m}$, seguidas por 1-2 células más cortas; los conidios fueron elipsoides-ovoides, cilíndricos o doliiformes, midiendo 23.5$42.7 \times 11.6-18.9 \mu \mathrm{m}$. No se observó la presencia de casmotecios. Estas características fueron similares a las descritas por Braun y Cook (2012) para Pseudoidium hortensiae. Este hongo se ha reportado ampliamente a nivel mundial infectando plantas de Hydrangea spp. (Farr y Rossman, 2018). En México, Yáñez-Morales et al. (2009) previamente identificaron mediante análisis en $\mathrm{ML}$ a $P$. hortensiae sobre Hydrangea sp. en muestras de Texcoco, Estado de México.

La examinación en $\mathrm{ML}$ del hongo sobre hojas de L. vulgare mostró conidióforos erectos, rectos y midiendo de 108 a $144 \mu \mathrm{m}$ de longitud (Figura 5A); las células base de los conidióforos fueron cilíndricas o incrementándose ligeramente en amplitud de la base a la punta de 58.6-84.6 × 10.9-14.4 $\mu \mathrm{m}$, seguidas por 1-2 células más cortas (Figura 5B); los conidios fueron catenescentes, elipsoides-ovoides a doliiformes, midiendo de 23.3-37.8 × 12.6-20.5 um (Figura 5C); los tubos germinativos fueron terminales y simples. Por otra parte, la examinación en MEB exhibió apresorios hifales solitarios y en forma de pezón (Figura 5D); conidióforos con conidios catenados (Figura 5E); los conidios turgentes presentaron pared externa con patrón granuloso y con
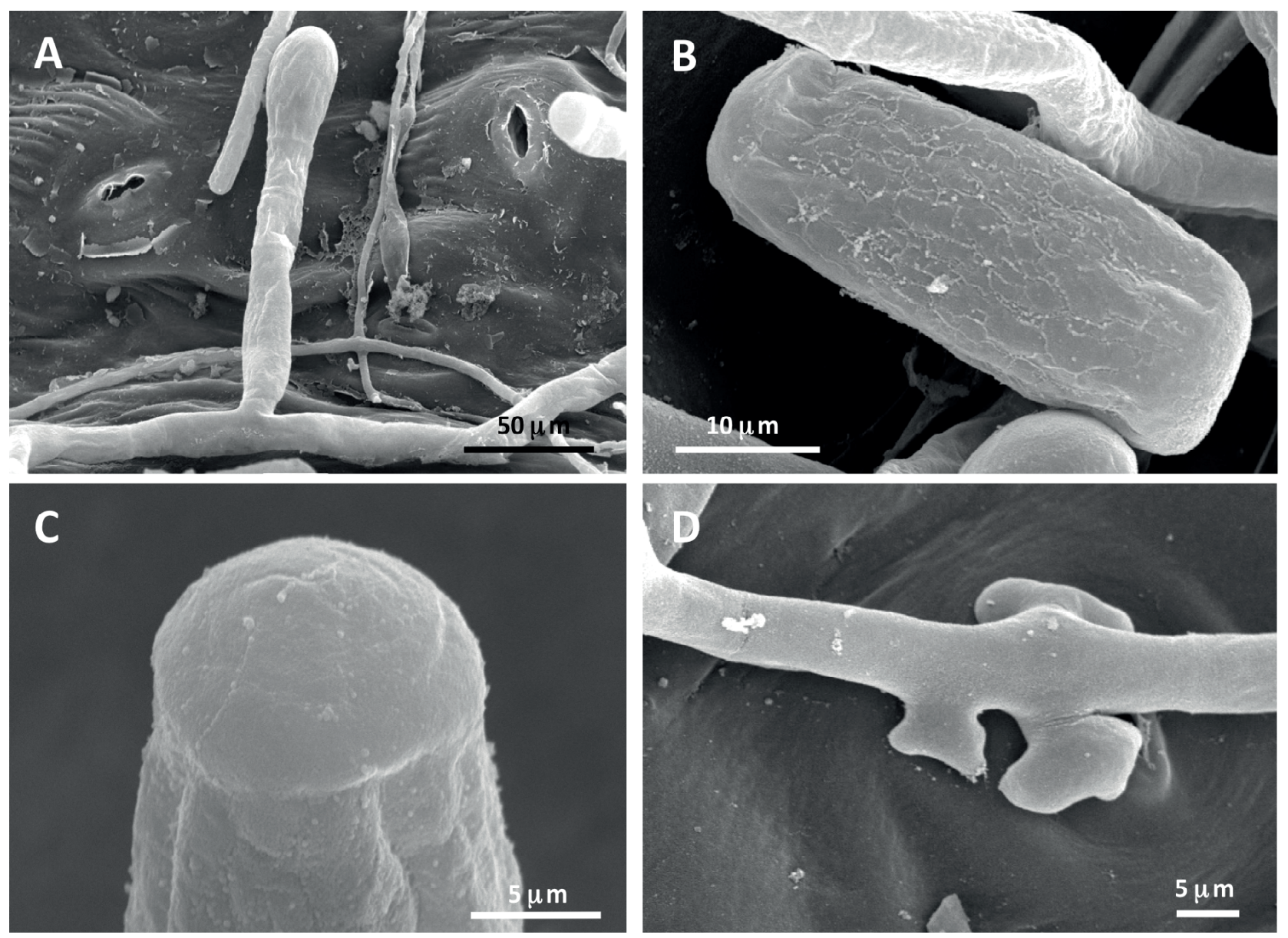

Figura 4. Micrografías en microscopia electrónica de barrido de Pseudoidium hortensiae. A) Conidióforo con conidio simple. B) Conidio turgente con superficie exterior con patrón rugoso. C) Conidio mostrando superficie de pared terminal con patrón fibrilar. D) Apresorios hifales multilobados solitarios y dispuestos en pares opuestos. 
superficie de la parte terminal con patrón fibrilar (Figura 5F), mientras que los conidios parcialmente deshidratados mostraron un patrón reticulado en la pared externa y un patrón fibrilar en la superficie de la parte terminal. No se observó la presencia de casmotecios. Con base en los caracteres morfológicos observados en ML y MEB, el hongo se identificó como el estado asexual de Golovinomyces macrocarpus (Braun y Cook, 2012). Para nuestro conocimiento, este es el primer reporte de G. macrocarpus causando cenicilla en L. vulgare en México. Este patógeno, se ha reportado sobre hospedantes de la familia Asteraceae en países
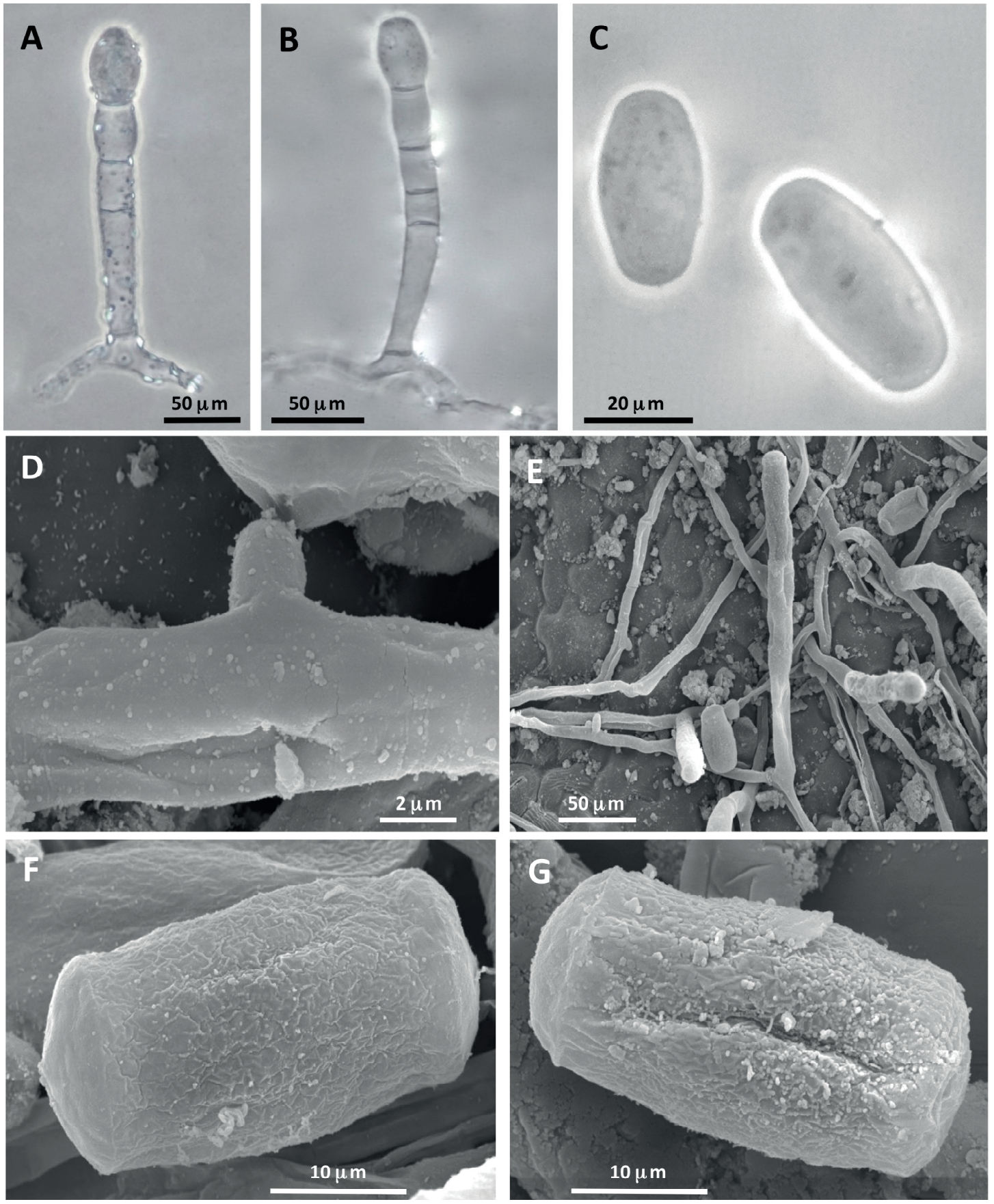

FIGURA 5. Micrografías en microscopia de luz (A-C) y microscopia electrónica de barrido (D-G) del estado asexual de Golovinomyces macrocarpus. A-B) Conidióforos con conidios simples. C) Conidios. D) Apresorio hifal solitario y en forma de pezón. E) Conidióforos con conidios en cadena. F) Conidio turgente con patrón superficial granuloso y patrón terminal fibrilar. G) Conidio parcialmente deshidratado con patrón superficial reticulado y patrón terminal fibrilar. 
de Europa, África del Norte (Marruecos), Norteamérica (Canadá y EE. UU.), Sudamérica (Argentina), y Asia (Irán, Japón, Kazakstán y Rusia) (Braun y Cook, 2012). La examinación en ML del hongo en C. limon mostró hifas hialinas, septadas, de 2.2-5.4 $\mu \mathrm{m}$ de ancho y con apresorios lobulados (2-3); los conidióforos fueron erectos y midiendo $29.9-62.1 \times 6.6-9.9 \mu \mathrm{m}$; las células basales fueron rectas, cilíndricas, de 23.3-36.6 $\times$ 6.5-9.8 $\mu \mathrm{m}$ (Figuras 6A-B) seguida por 1-2 células cortas que dan origen a un conidio simple; los conidios primarios fueron obovoides-elipsoidales, hialinos, con ápice redondeado y base subtruncada (Figura 6C); los conidios secundarios fueron doliiformes, hialinos, con los ápices subtruncados, de 20.9-32.5 × 10.8-14.7
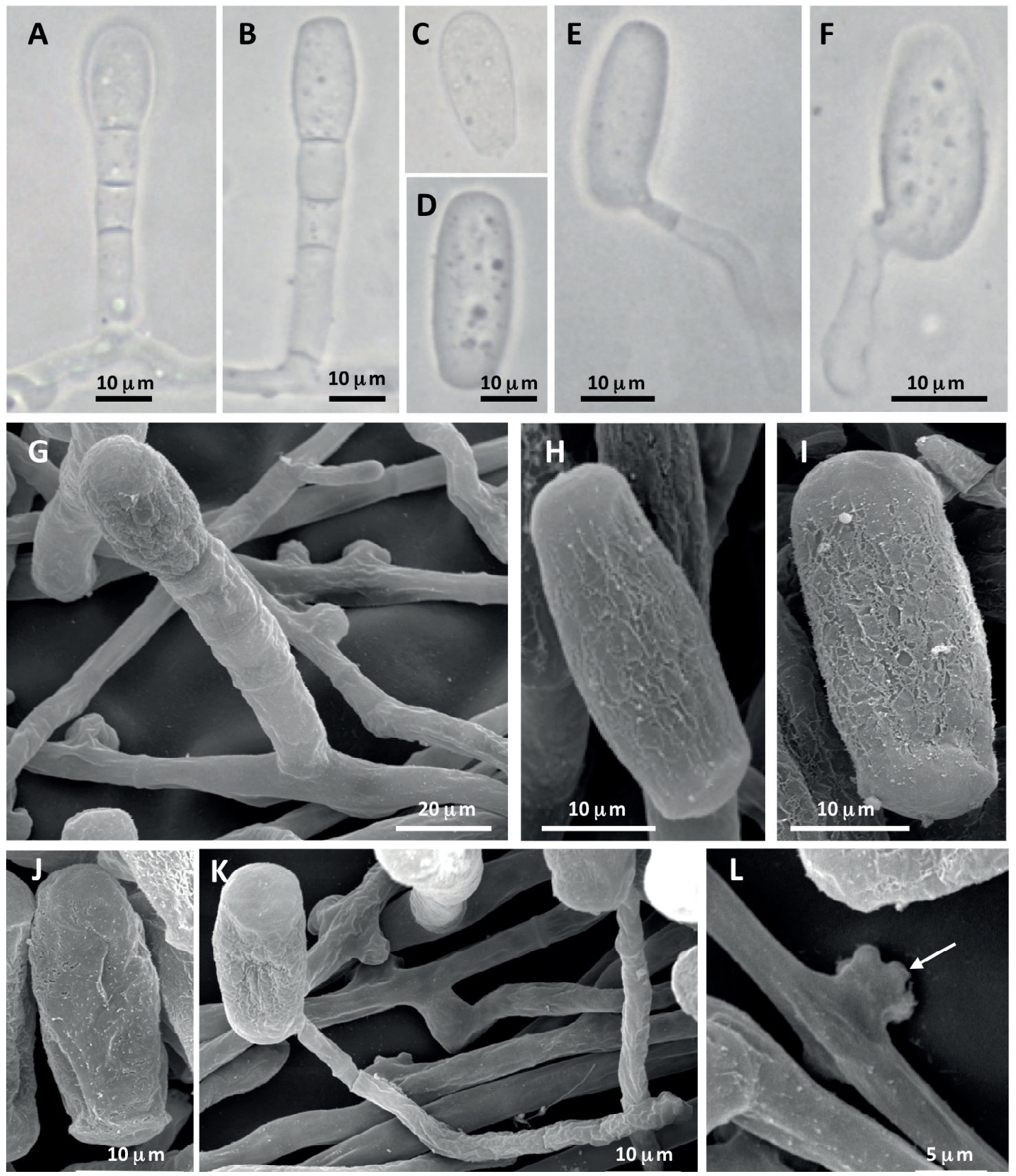

FiguRa 6. Micrografías en microscopia de luz (A-F) y microscopia electrónica de barrido (G-L) del estado asexual de Erysiphe quercicola. A) Conidióforo con conidio primario. B) Conidióforo con conidio secundario. C) Conidio primario. D) Conidio secundario. E-F) Conidios con tubo germinativo simple. G) Conidióforo con conidio turgente simple. $\mathrm{H}-\mathrm{I}$ ) Conidios secundarios turgentes con pared exterior con patrón rugoso. J) Conidio primario con pared externa con patrón rugoso. K) Conidio con pared terminal con patrón fibrilar y con tubo germinativo simple. L) Apresorio hifal multilobado. 
$\mu \mathrm{m}$ (Figura 6D); los tubos germinativos fueron simples (Figuras 6E-F). No se observaron casmotecios. Todas las características observadas en $\mathrm{ML}$ correspondieron con las reportadas por Braun y Cook (2012) para el estado asexual de Erysiphe quercicola. En un estudio previo llevado a cabo por Yáñez-Morales et al. (2009), se identificó mediante análisis en ML a E. quercicola infectando árboles de limón en muestras recolectadas en Tenancingo, Villa Guerrero y Texcoco en el Estado de México; en Ocuituco, Morelos; y en San Salvador el Verde, Puebla. Con respecto a MEB, se observaron conidióforos con conidios simples (Figura 6G); los conidios turgentes primarios (Figuras $6 \mathrm{H}-\mathrm{I}$ ) y secundarios (Figura 6J) mostraron pared externa con patrón rugoso, mientras que, la pared terminal presentó patrón superficial fibrilar (Figura 6K); los tubos germinativos fueron simples (Figura 6K) y los apresorios miceliales fueron multilobados (Figura 6L). De manera similar, Holford et al. (2010) identificaron a Oidium citri (sin. E. quercicola) en árboles de mandarina en Bután usando ML y MEB, asi como análisis de secuencias de la región de los espaciadores internos transcritos (ITS) del ADN ribosómico. No obstante, estos autores únicamente publicaron las micrografías, pero no describieron los patrones de la pared externa y pared terminal de conidios observados en MEB.

En general, las examinaciones en MEB realizadas en este estudio permitieron diferenciar a los géneros Erysiphe, Podosphaera, Pseudoidium y Golovinomyces con base en los patrones de las paredes externas y terminales de conidios. Esto confirmó lo indicado por Cook et al. (1997), Braun et al. (2002), y Braun y Cook (2012), quienes mencionaron que los patrones superficiales de conidios vistos en MEB, ayudan a la identificación precisa de los géneros de anamorfos de Erysiphales, permitiendo la identificación del género del teleomorfo en ausencia de su ascoma. Asimismo, se llevó a cabo la identificación a nivel de especie de cuatro Erysiphales mediante características morfológicas de conidióforos, conidios, apresorios hifales, tubos germinativos y apresorios conidiales vistas en ML y MEB.

En conclusión, este estudio muestra de manera detallada la examinación morfológica mediante MEB y $\mathrm{ML}$ de cinco especies de Erysiphales, incluyendo a Erysiphe sp., Podosphaera xanthii, Pseudoidium hortensiae, Golovinomyces macrocarpus y E. quercicola. Estos datos morfológicos servirán para ampliar el conocimiento sobre estudios de Erysiphales en México y a nivel mundial. Además, este estudio muestra el primer reporte de $G$. macrocarpus infectando L. vulgare en México.

\section{LITERATURA CITADA}

Bereczky, Z., A. Pintye, P. Csontos, U. Braun, L. Kiss, 2015. Does the parasite follow its host? Occurrence of morphologically barely distinguishable powdery mildew anamorphs on Oenothera spp. in different parts of the world. Mycoscience 56: 267-272.

Bozzola, J.J., L.D. Russell, 1992. Electron Microscopy: Principles and techniques for biologists. Jones and Bartlett Publishers, Boston, Massachusetts.

Bradshaw, M., U. Braun, M. Götz, J. Meeboon, S. Takamatsu, 2017. Powdery mildew of Chrysanthemum $\times$ morifolium: phylogeny and taxonomy in the context of Golovinomyces species on Asteraceae hosts. Mycologia 109(3): 508-519.

Braun, U., 1999. Some critical notes on the classification and the generic concept of the Erysiphaceae. Schlechtendalia 3: 48-54.

Braun, U., R.T.A. Cook, A.J. Inman, H.D. Shin, 2002. The Taxonomy of the powdery mildew fungi. In: Bélanger, R.R., W.R. Bushnell, A.J. Dik, T.L.W. Carver (eds.), The powdery mildews: A comprehensive treatise. American Phytopathological Society. St. Paul Minnesota. Pp. 13-55.

Braun, U., R.T.A. Cook, 2012. Taxonomic manual of the erysiphales (powdery mildews). CBS-KNAW Fungal Biodiversity Centre. Utrecht.

Camacho-Tapia, M., V. Sánchez-Soto, K.C. Correia, K. Pastirčáková, J. M. Tovar-Pedraza. 2018. Powdery mildew of California poppy caused by Erysiphe eschscholziae in Mexico. Canadian Journal of Plant Pathology 40: 461-466

Cook, R.T.A., A.J. Inman, C. Billings, 1997. Identification and classification of powdery mildew anamorphs using light and scanning electron microscopy and host range data. Mycological Research 101:975-1002.

Domínguez-Serrano, D., R. García-Velasco, M.E. Mora-Herrera, M.L. Salgado-Siclan, 2016. Identificación y alternativas de manejo de la cenicilla del rosal. Revista Mexicana de Fitopatología 34: 22-42.

Farr, D.F., A.Y. Rossman, 2018. Fungal databases, U.S. National Fungus Collections, ARS, USDA. Retrieved April 15, from https:// nt.ars-grin.gov/fungaldatabases/

Félix-Gastélum, R., M.A. Apodaca-Sánchez, M.C. Martínez-Valenzuela, S. Espinosa-Matías, 2005. Podosphaera (sect. Sphaerotheca) xanthii (Castagne) U. Brawn y N. Shishkoff en cucurbitáceas en el norte de Sinaloa, México. Revista Mexicana de Fitopatología 23: 162-168.

Félix-Gastélum, R., J.A. Ávila-Díaz, B.O. Valenzuela-Cota, J.A. Trigueros-Salmerón, R.M. Longoria-Espinoza, 2007. Identificación y control químico de los agentes causales de la mancha foliar y de cenicilla en tomatillo (Physalis ixocarpa Brot.) en el Norte de Sinaloa, México. Revista Mexicana de Fitopatología 25: 1-10.

Félix-Gastélum, R., I.E. Maldonado-Mendoza, G. Herrera-Rodríguez, C. Martínez-Valenzuela, S. Espinosa-Matías, J.D. Cordero-Ramírez, J.C. Martínez-Álvarez, 2011. Powdery mildew on common bean (Phaseolus vulgaris L.) in Northern Sinaloa, Mexico. Sydowia 63: 169-182. 
Félix-Gastélum, R., G. Herrera-Rodríguez, C. Martínez-Valenzuela, R.M. Longoria-Espinoza, I.E. Maldonado-Mendoza, F.R. Quiroz-Figueroa, J.C. Martínez-Álvarez, L.M. García-Pérez, S. Espinoza-Matías, 2013. First report of powdery mildew (Pseudoidium anacardii) of mango trees in Sinaloa, Mexico. Plant Disease 97: 994.

Félix-Gastélum, R., G. Herrera-Rodríguez, C. Martínez-Valenzuela, I.E. Maldonado-Mendoza, F.R. Quiroz-Figueroa, H. Brito-Vega, S. Espinosa-Matías, 2014. First report of powdery mildew (Podosphaera pannosa) of roses in Sinaloa, Mexico. Plant Disease 98(10):1442.

Garibaldi, A., G. Gilardi, M.L. Gullino, 2008. First report of powdery mildew caused by Podosphaera xanthii on Calendula officinalis in Italy. Plant Disease 92(1): 174.

Gleason, M.L., M.L. Daughtrey, A.R. Chase, G.W. Moorman, D.S. Mueller, 2009. Diseases of herbaceous perennials. APS Press, St. Paul, Minnesota.

Gorter, G.J.M.A., 1987. Relevance of conidial surface structure for identifying Erysiphe galeopsidis DC. South African Journal of Science 83: 112-114.

Hammett, K.R.W., 1977. Taxonomy of Erysiphaceae in New Zealand. New Zealand Journal of Botany 15: 687-711.

Holford, P., N.J. Donovan, Thinlay, E. Kabanoff, O. Wildman, S. Hardy, G.A.C. Beattie, N. Om, Dorjee, P. Wangdi, 2010. First report of Oidium citri in Bhutan. Australasian Plant Disease Notes 5: 55-57.
Lucero, G.S., P. Pizzuolo, M.L. López, 2007. Primera cita de Podosphaera fusca, responsable del oídio de la caléndula en Mendoza, Argentina. Revista FCA UNCuyo 39(1): 117-122.

Pastirčáková, K., T. Jankovics, J. Komáromi, A. Pintye, M. Pastirčák, 2016. Genetic diversity and host range of powdery mildews on Papaveraceae. Mycological Progress 15:36.

Plumb, R.T., R.H. Turner, 1972. Scanning electron microscopy of Erysiphe graminis. Transactions of the British Mycological Society 59: 149-178.

Scholler, M., A. Schmidt, S.A.S. Siahaan, S. Takamatsu, U. Braun, 2016. A taxonomic and phylogenetic study of the Golovinomyces biocellatus complex (Erysiphales, Ascomycota) using asexual state morphology and rDNA sequence data. Mycological Progress 15:56.

Tovar-Pedraza, J.M., S.G. Leyva-Mir, E.H. Nieto-López, C. Nava-Díaz, P.H. Goodwin, G.H. Rosas-Saito, 2016. First report of powdery mildew caused by Erysiphe heraclei on Ammi majus in Mexico. Plant Disease 100(3): 647.

Woodward, J.L.W., M.L. Daughtrey, 2001. Hydrangea diseases. In: Jones R.K., Benson D.M. (eds), Diseases of woody ornamentals and trees in nurseries. APS Press, St. Paul Minnesota. Pp. 191194.

Yáñez-Morales, M.J., U. Braun, A.M. Minnis, J.M. Tovar-Pedraza, 2009. Some new records and new species of powdery mildew fungi from Mexico. Schlechtendalia 19: 47-61. 